\title{
Investimento, internações e óbitos por câncer de boca na cidade de Feira de Santana, Bahia
}

\author{
Investment, hospitalizations and deaths \\ from mouth cancer in the city of Feira de \\ Santana, Bahia
}

\section{Igor Ferreira Borba de Almeida' 1 Deybson Borba de Almeida 2 (1)}

\begin{abstract}
'Autor para correspondência. Universidade Estadual de Feira de Santana (Feira de Santana). Bahia, Brasil. borbadealmeidaigor@gmail.com
\end{abstract} ${ }^{2}$ Universidade Estadual de Feira de Santana (Feira de Santana). Bahia, Brasil. dbalmeida@uefs.br

RESUMO | INTRODUÇÃO: O câncer de boca é uma doença crônica de alto custo social que exige para o seu diagnóstico e tratamento métodos especializados e internações hospitalares de alto custo. OBJETIVO: Descrever o investimento financeiro, frequência e tempo de permanência de internações e perfil dos óbitos por câncer de boca no município de Feira de Santana, estado da Bahia, no ano de 2020. MÉTODOS E MATERIAIS: Estudo do tipo descritivo que analisou os dados do Sistema de Informação Hospitalar do Sistema Único de Saúde (SIH/SUS) sobre os gastos públicos e privados, internações e óbitos decorrentes do câncer de lábio, boca e faringe no município de Feira de Santana, na Bahia, no período de janeiro a outubro de 2020. RESULTADOS: No período analisado, o município informou 206 internações por câncer de boca, tendo um total de 726 dias de permanência nos hospitais e um investimento total no valor de $\mathrm{R} \$ 421.019,42$. O sexo masculino $(85,7 \%)$, a cor parda $(64,2 \%)$ e a faixa etária de 30 a 59 anos $(57,1 \%)$ apresentaram as maiores frequências de óbitos devido à doença. A taxa de mortalidade para a cidade foi de 5,6. CONCLUSÃO: A análise deste estudo demonstrou que, no município de Feira de Santana, houve maior frequência de óbitos relativos ao câncer de boca em homens, adultos jovens e pardos. As internações decorrentes da doença somaram mais de 700 dias de permanência nos hospitais localizados no município, o que pode contribuir para elevação dos custos hospitalares.

PALAVRAS-CHAVE: Câncer de boca. Custos hospitalares. Taxa de mortalidade.

\begin{abstract}
INTRODUCTION: Oral cancer is a chronic disease with a high social cost that requires specialized methods and expensive hospitalizations for its diagnosis and treatment. OBJECTIVE: To describe the financial investment, frequency, and length of hospital stay and profile of deaths from oral cancer in the municipality of Feira de Santana, State of Bahia, in 2020. METHODS AND MATERIALS: A descriptive study that analyzed the data of the Hospital Information System of the Unified Health System (SIH / SUS) on public and private spending, hospitalizations, and deaths due to cancer of the lip, mouth, and pharynx in the municipality of Feira de Santana, Bahia, from January to October 2020. RESULTS: In the period analyzed, the municipality reported 206 admissions for oral cancer, with a total of 726 days of hospital stay, adding up to a total investment of $R \$ 421,019.42$. The male gender (85.7\%), the brown color (64.2\%), and the age group of 30 to 59 years (57.1\%) presented the highest frequency of deaths due to the disease. The death rate for the city was 5.6. CONCLUSION: The analysis of this study showed that, in the municipality of Feira de Santana, there was a higher frequency of deaths related to oral cancer in men, young adults, and browns. Hospitalizations due to the disease added up to more than 700 days of stay in hospitals located in the municipality, which can contribute to increased hospital costs.
\end{abstract}

KEYWORDS: Oral cancer. Hospital costs. Mortality rate. 


\section{Introdução}

Câncer é um termo que abrange mais de 100 diferentes tipos de doenças malignas que têm em comum o crescimento desordenado de células, que podem invadir tecidos adjacentes ou órgãos a distância. É considerado uma doença crônica de alto custo social que exige métodos especializados de diagnóstico por imagem, internação hospitalar e acompanhamento ambulatorial ${ }^{1}$.

No tocante à Epidemiologia, a Agência Internacional de Pesquisa em Câncer (IARC) estimou para o ano de 2020, aproximadamente 370.000 novos casos de câncer bucal, 97.000 de câncer de orofaringe e 240.000 mortes por essas neoplasias no mundo com uma incidência anual estimada em 274 mil novos casos e 128 mil óbitos $^{2}$. Para o Brasil, foram estimados para cada ano do triênio 2020-2022, 11.180 casos de câncer de boca em homens e 4.010 em mulheres ${ }^{1}$.

Apesar de ser uma doença genética, outros fatores são relacionados ao desenvolvimento das lesões. Nesse sentido, o tabaco e álcool são amplamente conhecidos como fatores promotores e potencializadores da doença ${ }^{3}$. Recentemente, o Papilomavírus Humano (HPV), acompanhado do comportamento sexual das populações, também está associado ao câncer de orofaringe ${ }^{4,5}$. A exposição solar, aumento da gordura corpórea e desordens potencialmente malignas também estão relacionados ao aparecimento deste tipo de câncer ${ }^{6}$.

Como não há um consenso na literatura nacional e internacional sobre quais localizações compõem a sua definição, foram consideradas como câncer da cavidade oral aquelas que tenham como localização primária lábios, cavidade oral, glândulas salivares e orofaringe e são classificadas com os códigos $\mathrm{COO}-\mathrm{C} 10^{1}$.

O tratamento dessas lesões envolve tecnologias especializadas de alto custo que contemplam ressecção cirúrgica, quimioterapia e radioterapia de forma isolada ou combinada o que pode comprometer a funcionalidade e qualidade de vida dos indivíduos, por este motivo o diagnóstico precoce e políticas de alerta para reduzir os fatores de risco são de fundamental importância para o prognóstico, aumento da taxa de sobrevida (atualmente de 5 anos) e redução da frequência dessas lesões ${ }^{\underline{7}, .}$.
Apesar do empenho dos programas de prevenção do câncer de boca do Sistema Único de Saúde, no Brasil, a maior parte dos pacientes é hospitalizada em estágios avançados da doença o que resulta em alta morbimortalidade. Além disso, ressalta-se que os estágios avançados resultam em pior prognóstico com tratamento mais agressivo, maior investimento público e privado e impactos negativos na qualidade de vida dos indivíduos acometidos?

Neste cenário, os estudos epidemiológicos contribuem significativamente para elucidar questões relevantes sobre a saúde das populações e, assim, servir de base para o desenvolvimento de políticas públicas eficazes garantindo equidade no cuidado. Desta forma, este artigo tem o objetivo de descrever o investimento financeiro, frequência e tempo de permanência de internações e perfil dos óbitos por câncer de boca no município de Feira de Santana, estado da Bahia, no ano de 2020.

\section{Métodos e Materiais}

Estudo do tipo descritivo que analisou os dados do Sistema de Informação Hospitalar do Sistema Único de Saúde (SIH/SUS) sobre os gastos públicos e privados, internações e óbitos decorrentes do câncer de lábio, boca e faringe no município de Feira de Santana, na Bahia. Esse sistema integra a base de dados do Departamento de Informação e Informática do SUS (DATASUS), plataforma de Informações de Saúde (TABNET) - tabulador de dados (http://www2. datasus. gov.br/DATASUS/index.php).

Os estudos descritivos têm por objetivo determinar a distribuição de doenças ou condições relacionadas à saúde, segundo o tempo, o lugar e/ou as características dos indivíduos, podendo utilizar dados secundários ou primários ${ }^{10}$.

O local de estudo, o município de Feira de Santana, está localizado na região metropolitana do estado da Bahia, com população estimada de 619.609 habitantes. A cidade é considerada um grande centro regional da área de saúde pois existem dezenas de hospitais públicos e privados ${ }^{1}$. Para adultos, o centro de referência para tratamento do câncer de boca e orofaringe é o Hospital Dom Pedro de Alcântara'. 
A análise dos dados ocorreu em janeiro de 2021, sendo considerado o período de janeiro a outubro de 2020 (período do ano disponível no DATASUS até o fechamento desta análise). Para compor a amostra, realizou-se a análise dos óbitos, utilizando-se as varáveis: sexo (masculino e feminino); idade; cor/raça, além disso obteve-se dados referentes às internações e valor total (gastos com serviços hospitalares e profissionais públicos e privados). Para esta análise, pesquisou-se a causa segundo a lista de morbidades da CID10, utilizando-se o capítulo II (neoplasias), especificando a Neoplasia maligna do lábio, cavidade oral e faringe (C00-C10).

Após obtenção dos dados, realizou-se a inserção em planilha do programa Microsoft Office Excel - 2010 para análise. Não houve necessidade de aprovação por um Comitê de Ética e Pesquisa por se tratar de um estudo que utiliza pesquisa em banco de dados, cujas informações são agregadas, sem possibilidade de identificação individual ${ }^{11}$.

\section{Resultados}

A análise e interpretação dos dados obtidos com esse estudo demonstrou que, em relação ao número de internações de pacientes com neoplasias maligna do lábio, cavidade oral e faringe, o município de Feira de Santana obteve 205 internações, totalizando 726 dias de permanência nos hospitais e um investimento de $R \$ 421.019,42$. Para o estado da Bahia, foram registradas 1.106 internações, totalizando 6.625 dias de permanência e um investimento de $\mathrm{R} \$ 45.148 .067$,44. Já no Brasil, foram registradas 20.169 internações, totalizando 107.932 dias de permanência dos hospitais, nos quais foram investidos $R \$ 45.148 .067$,44. (Tabela 1)

Tabela 1. Frequência de internações, dias de permanência e valor total gastos em reais, segundo lista de morbidade hospitalar (CID10 - Neoplasia maligna do lábio, cavidade oral e faringe), nos meses de janeiro a outubro de 2020, em Feira de Santana, na Bahia e no Brasil.

\begin{tabular}{cccc}
\hline Local & $\begin{array}{c}\text { Número de } \\
\text { internações }\end{array}$ & $\begin{array}{c}\text { Dias de } \\
\text { permanência }\end{array}$ & $\begin{array}{c}\text { Valor total dos gastos } \\
\text { em reais }\end{array}$ \\
\hline Feira de Santana & 205 & 726 & $\mathrm{R} \$ 421.019,42$ \\
Bahia & 1.106 & 6.625 & $\mathrm{R} \$ 2.543 .411,54$ \\
Brasil & 20.169 & 107.932 & $\mathrm{R} \$ 45.148 .067,44$ \\
\hline Fonte: Ministério da Saúde - Sistema de Informações Hospitalares do SUS (SIH/SUS)
\end{tabular}

No que diz respeito às características sociodemográficas dos óbitos de pacientes com este tipo de neoplasia, segundo a lista de morbidade hospitalar, no município de Feira de Santana, Bahia, a maior parte era do sexo masculino 12 (87,7\%); a cor parda foi a que mais prevaleceu $9(64,2 \%)$ com a faixa etária de 30 a 59 anos $(57,1 \%)$ tendo maior frequência. (Tabela 2) 
Tabela 2. Frequência e características sociodemográficas de óbitos, segundo lista de morbidade hospitalar (CID10 - Neoplasia maligna do lábio, cavidade oral e faringe, nos meses de janeiro a outubro de 2020, em Feira de Santana e na Bahia.

\begin{tabular}{|c|c|c|}
\hline Variáveis & Feira de Santana & Bahia \\
\hline & $\mathrm{n}(\%)$ & n (\%) \\
\hline \multicolumn{3}{|l|}{ Sexo } \\
\hline Masculino & $12(85,7 \%)$ & $114(71,6 \%)$ \\
\hline Feminino & $2(14,3 \%)$ & $45(28,4 \%)$ \\
\hline Total & 14 & 159 \\
\hline \multicolumn{3}{|l|}{ Raça ou cor } \\
\hline Branca & 0 & $14(8,8 \%)$ \\
\hline Preta & 0 & $18(11,3 \%)$ \\
\hline Parda & $9(64,2 \%)$ & $103(64,77 \%)$ \\
\hline Sem informação & $5(35,8 \%)$ & $24(15,1 \%)$ \\
\hline Total & 14 & 159 \\
\hline \multicolumn{3}{|l|}{ Faixa etária } \\
\hline 30 a 59 anos & $8(57,1 \%)$ & $76(47,7 \%)$ \\
\hline 60 anos ou mais & $6(42,9 \%)$ & $83(52,3 \%)$ \\
\hline Total & 14 & 159 \\
\hline
\end{tabular}

Fonte: Ministério da Saúde - Sistema de Informações Hospitalares

do SUS (SIH/SUS)

A taxa de mortalidade (cálculo: número de óbitos dividido pelo número de Autorização por Internações Hospitalares (AlH) pagas, computadas como internações, no período, multiplicando-se por 100), em Feira de Santana, foi de 5,6. Os comparativos entre o município em análise, o estado da Bahia, a região Nordeste e o Brasil estão apresentados na tabela 3.

Tabela 3. Taxa de mortalidade por neoplasias malignas de lábio, cavidade oral e faringe, no período de janeiro a outubro de 2020.

\begin{tabular}{|c|c|c|c|c|}
\hline \multirow[t]{2}{*}{ Variável } & \multicolumn{4}{|c|}{ Local } \\
\hline & Feira de Santana & Bahia & Região Nordeste & Brasil \\
\hline Taxa de mortalidade & 5,6 & 13,32 & 11,3 & 11,65 \\
\hline
\end{tabular}

\section{Discussão}

No período de análise deste estudo, o município de Feira de Santana, notificou 205 entradas para internações em seus hospitais públicos e privados, representando, portanto, 18,53\% do total de internações do estado da Bahia. A porcentagem relativamente alta, pode ser explicada porque o município é considerado grande centro regional da área de saúde ${ }^{12}$, desenvolvendo consórcios com diversas cidades vizinhas que não possuem serviços de atendimento locais para o câncer.

Com relação aos dias de permanência de internação, foram notificados 726 dias no total, representando 10,95\% dos dias, em todo o estado baiano. O valor total de Feira de Santana (equivalentes aos investimentos com serviços hospitalares e profissionais) dos gastos públicos e privados foi de $R \$ 421.019,42$, equivalendo a $16,55 \%$ dos gastos da Bahia e 0,93\% dos gastos de todo o Brasil. 
É importante considerar que a morbimortalidade por câncer vem aumentando expressivamente em todo o mundo, sobretudo devido ao envelhecimento populacional. Para o cenário brasileiro, as neoplasias malignas representam a segunda causa de morte, exigindo, portanto, medidas estratégicas para o seu controle, demandando dos gestores do Sistema Único de Saúde (SUS) enorme empenho para garantir oferta integral, equânime e satisfatória do cuidado em saúde ${ }^{1,13}$.

À medida que a população envelhece, os custos socioeconômicos associados às doenças crônicas não transmissíveis, incluindo, portanto, as neoplasias, originam impactos na economia mundial, sendo estimado um gasto de 7 trilhões de dólares, entre os anos de 2011-2025, nos países em desenvolvimento ${ }^{14}$. Corroborando o cenário mundial, estima-se para o Brasil, em 2030, uma taxa de mortalidade por câncer $45 \%$ maior que a atual, seguindo de uma população de idosos maior que a de jovens e crianças ${ }^{12}$.

A respeito dos gastos para o tratamento do câncer, deve-se considerar que é caracterizado como muito dispendioso e que nas últimas décadas o custo disparou, como resultado do aperfeiçoamento das técnicas de imagem, modernos equipamentos de radioterapia e agentes anticâncer de alto custo, incluindo terapias do tipo alvo-molecular, sobretudo nos países desenvolvidos ${ }^{15}$.

Os custos diretos com o tratamento do câncer de boca são relacionados ao diagnóstico e triagem, radioterapia, quimioterapia, cirurgia, manejo dos efeitos colaterais do tratamento e acompanhamento na sobrevida, sendo que o diagnóstico tardio eleva o custo, devido à metástase e recorrência, apesar da relevância e impacto financeiro, os estudos que avaliam os custos das internações hospitalares por câncer de boca são escassos na literatura ${ }^{16}$.

Nessa mesma perspectiva, é importante destacar que o número de tratamento e os gastos com câncer no Brasil aumentam substancialmente, mesmo sem correspondem às necessidades reais da população. Entre 1999 e 2015, os gastos somente com tratamento (principalmente para quimioterapia) passaram de $\mathrm{R} \$ 470$ milhões para $R \$ 3,3$ bilhões em valores nominais, ou seja, um crescimento de sete vezes num período de 16 anos $^{17}$.
Ressalta-se que os atuais parâmetros de tratamento, os custos do câncer aumentam exponencialmente, por ocorrerem em pacientes relativamente mais jovens, serem diagnosticados em estágios avançados, e necessitarem de terapias e tecnologias caras. A maior parte dos custos estão associados a tratamentos paliativos, onde as chances de cura em estágios avançados da doença já são muito improváveis. As estimativas da Sociedade Norte-Americana de Câncer consideram que os gastos com tratamento de todos os tipos de câncer, no mundo, aumentarão de US\$ 290 para US $\$ 458$ bilhões entre 2010 e $2030^{17}$.

Em países em desenvolvimento como o Brasil, por exemplo, dado que grande parte dos casos é detectada em estágios avançados e as políticas de prevenção do câncer ainda tem uma cobertura relativamente baixa, o número de pessoas que necessita de medicamentos e terapias (mesmo as novas e mais caras) tende a ser proporcionalmente elevado. Portanto, no ritmo atual de crescimento da doença, as necessidades de financiamento ficarão sempre aquém das possibilidades de atender a todas as necessidades ${ }^{17}$.

$\mathrm{Na}$ literatura nacional, apenas 4 estudos ${ }^{18-21}$ avaliaram tendência de óbitos por câncer de boca no país. Filho e Moncau ${ }^{19}$, em um estudo retrospectivo que avaliou os anos de 1980 a 1995, constataram que existe uma marcante heterogeneidade na mortalidade por câncer no Brasil, além disso, nesse período avaliado, ficou evidente a discreta queda no número de óbitos devido ao câncer de boca, lábio e orofaringe. Considerou-se que políticas de prevenção primária e secundária, bem como a melhoria na sobrevida de pacientes com o diagnóstico de câncer podem ter contribuído para a tendência à queda da mortalidade por câncer observada nas regiões Sul e Sudeste. Os autores destacam que é difícil mensurar tendências da mortalidade e a carga que representa o câncer em regiões com registros de morte com baixos padrões de qualidade. A diferença entre o número de mortes notificadas e o número de mortes estimadas, situação que pode interferir na avaliação da magnitude do câncer em uma população particular, foi na região Norte, $40 \%$ menor do que a estimada. No Nordeste, esta diferença foi ainda maior, atingindo $45 \%$. 
Perea et al. ${ }^{21}$ estudaram a tendência de mortalidade por câncer de boca e faringe no Brasil, no período de 2002 a 2013 e concluíram que o coeficiente médio de mortalidade por câncer de boca foi de 1,87/100.00 habitantes e permaneceu estável durante o período estudado. Ressalta-se que a região Nordeste apresentou aumento de número de óbitos.

A respeito dos óbitos, nos países em desenvolvimento, como o Brasil, a taxa de sobrevida do câncer de boca é de cinco anos, estimada em 50 a $60 \%$ dos pacientes tratados. A taxa de sobrevida baixa está associada ao tipo celular mais agressivo das lesões e ao diagnóstico tardio ${ }^{20}$. Segundo pesquisa financiada ${ }^{20}$ pelo Tribunal de Contas da União em 2010, 60,5\% dos casos de câncer são diagnosticados no país nos estágios 3 e 4. Nestes estágios, os custos de tratamento costumam ser entre $60 \%$ e $80 \%$ maiores que nos estágios 1 e 2 , com possibilidades de cura sensivelmente menores ${ }^{17}$.

Neste estudo, o perfil epidemiológico dos óbitos por câncer de boca (que envolve lábio, boca e faringe) demonstrou que, em relação ao sexo, os homens morrem mais por esse tipo de doença, representando mais de $85 \%$ dos casos. Corroboram com esses resultados, os números da Bahia, que corresponderam $72 \%$ das mortes para o sexo masculino. Além disso, há consonância com os dados do INCA (2020)ㄹ, que considerou os homens com o triplo de acometimento em relação às mulheres. Corroborando os resultados deste estudo, Aquino et al. 22 também constataram que mais de $75 \%$ dos óbitos eram de homens. Em concordância, um estudo que analisou retrospectivamente 19 anos, de um serviço de patologia no estado da Bahia, constatou, também, que o sexo masculino foi o mais diagnosticado pelas neoplasias bucais $(61,94 \%)$ e a faixa etária mais acometida foi a de 25 a 64 anos $(23 \%)^{23}$.

Um estudo de série histórica de óbitos no município de Juiz de Fora, entre os anos de 1980 e 2005, concluiu que o risco de morrer foi maior entre homens do que entre mulheres para todas as localizações analisadas (boca, faringe e o conjunto boca e faringe), entretanto, chamou-se atenção a expressiva variação absoluta percentual no período para a mortalidade entre mulheres para o conjunto de tumores de cavidade oral e faringe. Uma das pressuposições para o avanço no comprometimento de mulheres por esse tipo de tumor refere-se ao aumento do tabagismo entre as mesmas ao longo dos anos. Essas mudanças sociais e comportamentais datam das décadas de 1960 e 1970 do século passado e são refletidas no aumento nas taxas de mortalidade por doenças ligadas ao tabagismo, como o câncer de pulmão e doenças cardiovasculares 24 .

Com relação à raça/cor dos indivíduos que morreram devido à doença, constatou-se que dos 14 (64,2\%) óbitos, 9 eram da cor parda e 5 não obtiveram informações. Para o estado da Bahia, do total de 159 óbitos, $103(64,77 \%)$ foram pardas, entretanto, a cor branca apresentou os menores números 14 (8,8\%). Aquino et al. ${ }^{22}$ também constataram maior prevalência de mortes em indivíduos pardos e pretos (62, 07\%). Em contrapartida aos achados no presente estudo, os autores Vasconcelos et al. $\underline{6}$ avaliaram 472 indivíduos e constataram que apesar de a maioria ser do sexo masculino $(62,71 \%)$, aproximadamente $60 \%$ dos indivíduos com câncer de boca eram leucodermas.

A distribuição étnico-racial da população brasileira no território nacional é também um aspecto que deve ser considerado nas análises de tendência por raça/ cor. Essa distribuição não é uniforme no Brasil; nas regiões Sul e Sudeste, predomina a população de raça/cor branca (78 e 55\% da população, respectivamente), enquanto nas regiões Norte e Nordeste, a raça/cor preponderante é a parda (67 e 59\% da população, respectivamente $)^{12}$.

A respeito da faixa etária, este estudo constatou que a faixa etária de 30 a 59 anos apresentou $8(57,1 \%)$ dos 14 óbitos, a faixa etária acima dos 60 anos apresentou $6(42,9 \%)$. Esses resultados estão seguindo a tendência do estado da Bahia, que para a primeira faixa etária apresentou 76 (47,7\%). A ocorrência de HPV em homens mais jovens, associada ao comportamento sexual, pode explicar a alta prevalência dos óbitos por esta doença ${ }^{14}$. Além disso, considerando os atuais parâmetros de tratamento, os custos do câncer aumentam exponencialmente, por ocorrerem em idades relativamente jovens, serem detectados tardiamente, e necessitarem de medicamentos e tecnologias caras $^{17}$. 
Com relação à taxa de mortalidade, a análise dos dados identificou que para o município de Feira de Santana foi de 5,6, para a Bahia 13,32, região Nordeste 13,3 e para o Brasil foi de 11,65. Esse índice leva em consideração o número de óbitos dividido pelo número de Autorização por Internações Hospitalares (AlH) pagas. As taxas de mortalidade têm sido tradicionalmente o indicador mais usado para diagnosticar a situação de saúde, porque é fácil calculá-las, por razões conceituais (a morte não se presta a ambiguidade) e funcional: preocupação com grupos específicos 25 .

Este estudo apresenta como principal característica a obtenção de dados secundários. Dessa forma, há limitações em função dos tipos de variáveis disponibilizadas, a existência de sub registros e a baixa qualidade de dados em algumas variáveis.

\section{Conclusão}

Neste estudo, foi observada maior frequência de óbitos por câncer de boca, no município de Feira de Santana, na Bahia, em homens relativamente jovens, com idade entre 30 e 59 anos e de cor parda. As internações devido à doença somaram mais de 700 dias de permanência nos hospitais localizados no município, o que pode contribuir para elevação dos custos hospitalares.

Deve-se considerar que o diagnóstico precoce e monitoramento das lesões diagnosticadas são de extrema importância para aumentar as taxas de sobrevida dos indivíduos comprometidos, oferecendo tratamentos menos invasivos e incapacitantes, além de reduzir impactos financeiro ao sistema de saúde.

Como perspectivas futuras, deve-se investir em estudos que subsidiem políticas específicas de prevenção e promoção da saúde bucal. Além disso, estudos que avaliem os custos como o tratamento desta doença é de fundamental importância para o planejamento efetivo e equânime do Sistema Único de Saúde.

\section{Contribuições dos autores}

Almeida IFB participou da concepção, delineamento, coleta e análise dos dados e escrita do manuscrito. Almeida DB participou da concepção, delineamento, análise dos dados e escrita do manuscrito.

\section{Conflitos de interesses}

Nenhum conflito financeiro, legal ou político envolvendo terceiros (governo, empresas e fundações privadas, etc.) foi declarado para nenhum aspecto do trabalho submetido (incluindo, mas não se limitando a subvenções e financiamentos, participação em conselho consultivo, desenho de estudo, preparação de manuscrito, análise estatística, etc.).

\section{Referências}

1. Instituto Nacional de Câncer José Alencar Gomes da Silva. Estimativa 2020: incidência de câncer no Brasil [Internet]. Rio de Janeiro: INCA; 2019 [acesso 2020 mar 7]. Disponível em: https:// www.inca.gov.br/sites/ufu.sti.inca.local/files/media/document/ estimativa-2020-incidencia-de-cancer-no-brasil.pdf

2. International Agency for Research on Cancer. Cancer Tomorrow [Internet]. 2018. Disponível em: http://gco.iarc.fr/tomorrow/ graphic-bar?type $=1 \&$ population $=900 \&$ mode $=$ population $\&$ sex $=0 \&$ cancer=39\&age_group=value \&apc_male $=0 \& a p c \_$female $=0 » h$ ttp: $/ /$ gco.iarc.fr/tomorrow/graphic-bar?type $=1 \&$ population $=900 \& \mathrm{~m}$ ode=population\&sex=0\&cancer $=39 \&$ \&age_group=value\&apc male $=0 \& a p c$ female $=0$

3. Huber MA, Tantiwongkosi B. Oral and Oropharyngeal Cancer. Med Clin North Am. 2014;98(6):1299-321. Citado em: PMID: $\underline{25443678}$

4. Hobbs CGL, Sterne JAC, Bailey M, Heyderman RS, Birchall MA, Thomas SJ. Human papillomavirus and head and neck cancer: a systematic review and meta-analysis. Clin Otolaryngol. 2006;31(4):259-66. Citado em: PMID: 16911640

5. Marur S, D'Souza G, Westra WH, Forastiere AA. HPV-associated head and neck cancer: A virus-related cancer epidemic. Lancet Oncol. 2010;11(8):781-9. https://doi 10.1016/S14702045(10)70017-6

6. Vasconcelos RM, Trindade JSO, Almeida ICP, Silva RJC, Morais MLSA. Perfil clínico-epidemiológico de câncer de boca em idosos. Rev. Gest. Saúde [Internet]. 2013;1:1984-91. https://periodicos. unb.br/index.php/rgs/article/download/214/203/438 
7. Leite MAC, Nogueira DA, Terra FS. Avaliação da autoestima em pacientes oncológicos submetidos a tratamento quimioterápico. Rev Latino-Am Enfermagem. 2015;23(6):1082-9. https://doi. org/10.1590/0104- 1169.0575.2652

8. Hunter KU, Jolly S. Clinical review of physical activity and functional considerations in head and neck cancer patients. Support Care Cancer. 2013;21:1475-9. https://doi.org/10.1007/ s00520-013-1736-4

9. Carvalho SHG, Soares MSM, Figueiredo RLQ. Levantamento epidemiológico dos casos de câncer de boca em um hospital de referência em Campina Grande, Paraíba Brasil. Pesq Bras Odontoped Clin Integr. 2012;12(1):47-51. https://doi: 10.4034/ PBOCI.2012.121.07

10. Lima-Costa MF, Barreto SM. Tipos de estudos epidemiológicos: conceitos básicos e aplicações na área do envelhecimento. Epidemiol Serv Saúde [Internet]. 2003;12(4): 89-201. Disponível em: http://scielo.iec.gov.br/scielo.php?script=sci arttext\&pid=S1679-49742003000400003

11. Resolução $n^{\circ}$ 510, de 07 de abril de 2016 (Brasil). Dispõe sobre as normas aplicáveis a pesquisas em Ciências Humanas e Sociais. [Internet]. Diário Oficial [da] República Federativa do Brasil. 2016 maio 24 [citado em 2020 jan. 04]. Disponível em: http://bit. ly/2fmnKeD

12. Instituto Brasileiro de Geografia e Estatística. [citado em 2020 ago 29]. Disponível em: https://www.ibge.gov.br/pt/inicio.html

13. Oliveira EXG, Melo ECP, Pinheiro RS, Noronha CP, Carvalho MS. Acesso à assistência oncológica: mapeamento dos fluxos origemdestino das internações e dos atendimentos ambulatoriais. 0 caso do câncer de mama. Cad Saúde Pública. 2011;27(2):317-26. http://doi.org/10.1590/S0102-311X2011000200013

14. Malta DC, Stopa SR, Santos MAS, Andrade SSCA, Oliveira TP, Cristo EB, et al. Evolução de indicadores do tabagismo segundo inquéritos de telefone, 2006-2014. Cad Saúde Pública [Internet]. 2017;33(3):e00134915. Disponível em: https://www.scielo.br/pdf/ csp/v33s3/1678-4464-csp-33-s3-e00134915.pdf

15. Jemal $A$, Vineis $P$, Bray FTL, editores. The Cancer Atlas. 2a. ed. Atlanta (EUA): American Cancer Society; 2014.

16. Wissinger E. The Economic Burden of Head and Neck Cancer: A Systematic Literature Review. PharmacoEconomics. 2014;32(9):865-82. http://doi.org/10.1007/s40273-014-0169-3
17. Medici AC. Custos do Tratamento do Câncer no Brasil: Como Melhorar o Foco. Portal Hospitais Brasil [Internet]. Portal Hospitais Brasil. Disponível em: https://portalhospitaisbrasil. com.br/artigo-custos-do-tratamento-do-cancer-no-brasil-comomelhorar-o-focol

18. Cunha AR, Prass TS, Hugo FN. Mortalidade por câncer bucal e de orofaringe no Brasil, de 2000 a 2013: tendências por estratos sociodemográficos. Ciênc. Saúde Coletiva. 2020;25(8):3075-86. https://doi.org/10.1590/1413-81232020258.31282018

19. Wünsch Filho V, Moncau JE. Mortalidade por câncer no Brasil 1980-1995: padrões regionais e tendências temporais. Rev. Assoc. Med. Bras. 2002;48(3):250-7. http://dx.doi.org/10.1590/S010442302002000300040

20. Bonfante GMS. Sobrevida de cinco anos e fatores associados ao câncer de boca para pacientes em tratamento oncológico ambulatorial pelo Sistema Único de Saúde, Brasil. Cad Saúde Pública. 2014;30(5):983-97. http://dx.doi.org/10.1590/0102$311 \times 00182712$

21. Perea LME, Peres MA, Boing AF, Antunes JLF. Tendência de mortalidade por câncer de boca e faringe no Brasil no período 2002-2013. R Saúde Pública. 2018;52:10. http://dx.doi. org/10.11606/s1518-8787.2018052000251

22. Aquino RCA, Lima MLLT, Menezes CRCX, Rodrigues M. Aspectos epidemiológicos da mortalidade por câncer de boca: conhecendo os riscos para possibilitar a detecção precoce das alterações na comunicação. Rev CEFAC. 2015;17(4):1254-61. https://doi.org/10.1590/1982-0216201517414914

23. Medrado AP, Assis MSA, Reis SRA. Occurrence of oral neoplasms and dysplastic lesions: retrospective study of a pathology service over a 19-years period. J Dent Public Health. 2020;11(2):111-120. http://dx.doi.org/10.17267/25963368dentistry. v11v2.3249

24. Hirota SK, Braga FP, Penha SS, Sugaya NN, Migliari DA. Risk factors for oral squamous cell carcinoma in young and older Brazilian patients: a compartive analysis. Med Oral Patol Oral Cir Bucal. 2008;13(4):227-31. Citado em: PMID: 18379445

25. Cardim MS, Azevedo BA, Morgado AF. O que a epidemiologia pode ainda fazer de relevante? Cad. Saúde Pública. 1991;7(1):6-16. http://dx.doi.org/10.1590/S0102-311X1991000100002 